\title{
MST4 promotes hepatocellular carcinoma epithelial-mesenchymal transition and metastasis via activation of the p-ERK pathway
}

\author{
ZHEN-HAI LIN $^{1 *}$, LIYING WANG $^{2 *}$, JU-BO ZHANG $^{1 *}$, YANFENG LIU $^{3}$, XIAO-QIANG LI ${ }^{1}$, \\ LEI GUO $^{1}$, BO ZHANG ${ }^{1}$, WEN-WEI ZHU ${ }^{1}$ and QING-HAI YE ${ }^{1}$ \\ ${ }^{1}$ Liver Cancer Institute and Zhongshan Hospital, Fudan University, and Key Laboratory of Carcinogenesis \\ and Cancer Invasion, Ministry of Education, Shanghai; ${ }^{2}$ The Second Artillery General Hospital of Chinese \\ People's Liberation Army, Beijing; ${ }^{3}$ Key Laboratory of Medical Molecular Virology (MOE and MOH), \\ Institute of Biomedical Sciences, Shanghai Medical College, Fudan University, Shanghai, P.R. China
}

Received February 23, 2014; Accepted April 24, 2014

DOI: $10.3892 / \mathrm{ijo} .2014 .2455$

\begin{abstract}
Mammalian sterile-20-like kinase 4 (MST4) has been implicated in cell proliferation and differentiation. In a previous study, we found MST4 to be an important candidate gene for metastatic hepatocellular carcinoma (HCC); however, the molecular mechanism of the promoting role of MST4 in HCC metastasis is poorly understood. In this study, we show that high expression of MST4 was detected in highly invasive HCC cells and in human HCC specimens with vascular invasion. A high level of MST4, associated with large tumor size, microvascular invasion, presence of intrahepatic metastasis, and advanced TNM classification of malignant tumors stage, was an independent prognostic factor for overall survival $(\mathrm{P}=0.004)$ and time to recurrence $(\mathrm{P}=0.001)$ after hepatectomy. Knockdown of MST4 expression in HCC cells inhibited cell proliferation, colony formation, and invasion, whereas upregulation of MST4 significantly promoted these processes by promoting epithelial-mesenchymal transition (EMT), dependent on the activation of extracellular signal-regulated protein kinase (ERK) signaling pathways. Furthermore, the combination of MST4 and phosphorylated ERK was proven to have more power to predict the outcomes of HCC patients. This study presents clinical evidence for predicting the value of MST4 in HCC overall survival and recurrence and describes the key role of MST4 in facilitating the EMT process via regulating the activation of ERK, indicating its potential role as a target for postoperative adjuvant therapy for HCC.
\end{abstract}

Correspondence to: Dr Qing-Hai Ye, Liver Cancer Institute and Zhongshan Hospital, Fudan University, 180 Feng Lin Road, Shanghai 200032, P.R. China

E-mail: ye.qinghai@zs-hospital.sh.cn

${ }^{*}$ Contributed equally

Key words: hepatocellular carcinoma, MST4, metastasis, recurrence, epithelial-mesenchymal transition, overall survival

\section{Introduction}

Hepatocellular carcinoma (HCC) is one of the most common malignancies and the third major cause of cancer death worldwide $(1,2)$. Despite the recent advances in diagnosis and treatment, the general prognosis of HCC still remains extremely dismal because of high postoperative metastatic recurrence (3). Increasing evidence suggests that epithelialmesenchymal transition (EMT) represents one important source of HCC cells with highly invasive capability (4).

EMT is a biological conversion process that transforms polarized epithelial cells to the mesenchymal phenotype $(5,6)$. The completion of EMT is signaled by the loss of cell-cell adhesion, the degradation of the underlying basement membrane, and the acquisition of migratory or invasive properties $(5,6)$. The molecular hallmark of EMT is the loss of epithelial marker E-cadherin and upregulation of the mesenchymal markers Vimentin, Snail, Slug, and others. In many cases, the involved factors are also used as biomarkers to demonstrate the passage of a cell through EMT (7). Multiple signaling pathways regulate the EMT process, which includes extracellular signal-regulated protein kinases (ERKs), mitogen-activated protein kinase (MAPK), phosphatidylinositol 3-kinase (PI3K)/ Akt, Smads, RhoB, and $\beta$-catenin signaling (8). Identification of the key molecules and the signaling pathways that lead to activation of EMT programs during this disease process provides new insights into the plasticity of cellular phenotypes and possible therapeutic interventions.

Mammalian sterile-20-like kinase 4 (MST4) is an important member of the GCK (also known as MAP4K2) subfamily (9) and belongs to the mammalian sterile-20 (Ste20)-like (MST) kinases (10), which play multiple roles in the regulation of signaling pathways governing cell mitosis, homeostasis, polarity, migration, apoptosis, proliferation, and differentiation (9-15). MST4 has low expression in the normal liver (16-18) and in some hepatoma cell lines such as PLC5 (16), whereas it is highly expressed in other hepatoma cells, such as SK-Hep1 (16). Studies imply that the expression of MST4 in different types of hepatoma cell lines is in disagreement. Overexpression of MST4 enhances ERK activity, leading to increased cell proliferation and cellular transformation 
$(19,20)$. Active MST4 can also stabilize to bind with GM130 (a cis-Golgi matrix protein) and regulate cell migration and polarization (21). In prostate cancer, MST4 accelerates the proliferation of prostate cancer cells and enhances anchorageindependent growth (20). In a previous study, we analyzed the expression profiles of HCCs without or with intrahepatic metastasis and found MST4 to be an important candidate gene for metastatic HCC (22). Based on these studies, we speculate that MST4 is a promoter of tumor progression and metastasis. However, little is known about the expression of MST4 in HCC samples and its role in the prognosis of HCC.

The present study shows that the high expression of MST4 is detected in highly invasive HCC cells and human HCC specimens with vascular invasion. Furthermore, Kaplan-Meier analysis suggests that high MST4 levels are associated with significantly worse clinical outcomes after surgical excision for HCC. Upregulation of MST4 in vitro significantly promotes cell proliferation, colony formation, and invasion of HCC by promoting EMT through the activation of ERK signaling pathways. The combination of MST4 and phosphorylated ERK (p-ERK) has better power to predict the outcomes of HCC. We report for the first time, that MST4 is a pivotal factor that facilitates HCC migration and metastasis and may become a potential adjuvant treatment target for aggressive HCC.

\section{Materials and methods}

Patients and clinical samples. A total of 178 primary HCC samples were randomly selected from patients who received curative resection for $\mathrm{HCC}$ at the Liver Cancer Institute and Zhongshan Hospital, Fudan University (Shanghai, China) from February 2006 to November 2006. This study was approved by the Zhongshan Hospital Research Ethics Committee. Follow-up procedures have been described previously (23). Each patient was followed-up until March 2012, with a median follow-up of 56 months (range, 2-69 months). The clinical characteristics of HCC patients are presented in Table I.

Tissue microarray and immunohistochemistry. Preparation of tissue microarray and immunohistochemistry procedures were performed as described (23). Antibodies used were anti-human MST4 (rabbit, polyclonal, 1:100; Proteintech) and anti-human p-ERK (rabbit, polyclonal, 1:200; Cell Signaling Technology). Two independent pathologists who did not have information regarding the patient characteristics assessed the immunohistochemistry staining. The immunohistochemistry staining intensity was graded on a scale from 0 to $3(0$, no staining; 1 , weak staining; 2 , mild staining; 3 , strong staining). The staining extent was graded on a scale from 0 to 3 based on the percentage of immunoreactive tumor cells $(0 \% ; 1-25 \%$; $26-50 \% ; 51-100 \%)$. The final score was obtained by multiplying the staining extent score by the staining intensity score. If the final score was $<3$, then it was defined as low expression. If the final score was $>3$, then it was defined as high expression.

Plasmid constructs. Flag-tagged and HA-tagged full-length MST4 cDNA were cloned in pcDNA3 (Invitrogen) to create pFlag-MST4 and pHA-MST4, respectively. Lentiviral vectors pLKO.1 TRC (Addgene plasmid 10879) and pWPI.1 (Addgene plasmid 12254) were used for producing recombinant lentivi- ruses. For RNA interference of MST4, DNA fragments encoding hairpin precursors for shMST4 group 1 (5'-CCTCTTGGTGTAT AGTATTTA-3') and shMST4 group 2 (5'-CCAGATTGCTA CCATGCTAAA-3') were inserted into pLKO.1 TRC, respectively. A scrambled siRNA precursor (Scr) of similar GC content as shMST4 group 1 and shMST4 group 2, but with no sequence identity with MST4, was used as a negative control. For overexpression of MST4, flag-tagged MST4 cDNA was cloned in pWPI.1.

Cell lines, transfection, and lentiviruses. The human hepatic non-tumor cell line LO2, the embryonic kidney cell line HEK293T, and HCC cell lines SMMC7721, PLC, and Hep3B were obtained from Cell Bank of Shanghai Institutes of Biological Sciences, Chinese Academy of Sciences (Shanghai, China). MHCC-97H and MHCC-LM3 cell lines were established at the Liver Cancer Institute and Zhongshan Hospital, Fudan University. All cells were cultured in DMEM highglucose supplemented with $10 \%$ fetal bovine serum (FBS) and $1 \%$ penicillin-streptomycin at $37^{\circ} \mathrm{C}$ with $5 \% \mathrm{CO}_{2}$.

The expression plasmids were transfected into cells using Lipofectamine 2000 (Invitrogen) according to the manufacturer's instructions. Helper plasmids pSPAX2 (Addgene plasmid 12260) and pMD2.G (Addgene plasmid 12259) were cotransfected with pLKO.1-based or pWPI.1-based plasmids in HEK293T cells to package recombinant lentiviruses. Supernatants from cotransfections were used for infection of cultured cells.

Quantitative reverse-transcription polymerase chain reaction. The detected cells were harvested in 6-well plates. The total RNA of each group was isolated using TRIzol (Gibco Laboratories). Reverse-transcription was performed with a PrimeScript reverse-transcription reagent kit (Takara Biotechnology, Dalian, China). Gene-specific primers for human MST4 and GAPDH were generated by Sangon Biotech (Shanghai, China). The following primer sequences were used: MST4: F-5'-TTCGAGCTGGTCCATTTGATG-3', R-5'-TGA ATGCAGATAGTCCAGACCT-3'; and GAPDH: F-5'-TGTG GGCATCAATGGATTTGG-3', R-5'-ACACCATGTATTCCG GGTCAAT-3'. The iCycler and iQ real-time polymerase chain reaction (Bio-Rad, Hercules, CA, USA) and SYBR-Green PCR Master Mix (Toyobo, Osaka, Japan) were used for amplification and detection. The steps of amplification were $95^{\circ} \mathrm{C}$ for $30 \mathrm{sec}, 60^{\circ} \mathrm{C}$ for $30 \mathrm{se}$ and $72^{\circ} \mathrm{C}$ for $30 \mathrm{sec}$ for 40 cycles. The data were analyzed by using the real-time polymerase chain reaction analysis software Bio-Rad iQ5. All experiments were performed in triplicate.

Cell proliferation assay. Cell proliferation was measured by Cell Counting Kit-8 (Dojindo, Kumamoto, Japan). According to the instructions, cultured cells were seeded onto 96-well plates at $5 \times 10^{3}$ per well ( $\mathrm{n}=5$ for each time-point) in a final volume of $100 \mu \mathrm{l}$. At indicated time-points, CCK-8 solution $(10 \mu \mathrm{l})$ was added to each well and the absorbance at $450 \mathrm{~nm}$ was monitored with Tecan Infinite M200 NanoQuant Daily (Mannedorf, Switzerland).

Colony formation assay. To detect clonogenesis ability, the cells $\left(1 \times 10^{3}\right.$ cells) were suspended with DMEM containing 
Table I. Comparison of clinicopathologic profiles between HCC patients with high MST4 and low MST4 levels.

\begin{tabular}{|c|c|c|c|c|c|}
\hline \multirow[b]{3}{*}{ Variables } & \multicolumn{4}{|c|}{ MST4 level } & \multirow[b]{3}{*}{ P-value } \\
\hline & \multicolumn{2}{|c|}{ High $(n=61)$} & \multicolumn{2}{|c|}{ Low $(n=117)$} & \\
\hline & Patients, $\mathrm{n}$ & $\%$ & Patients, $\mathrm{n}$ & $\%$ & \\
\hline \multicolumn{6}{|l|}{ Age, years } \\
\hline$\leq 50$ & 20 & 32.8 & 46 & 39.3 & 0.418 \\
\hline$>50$ & 41 & 67.2 & 71 & 60.7 & \\
\hline \multicolumn{6}{|l|}{ Sex } \\
\hline Female & 8 & 13.1 & 26 & 22.2 & 0.164 \\
\hline Male & 53 & 86.9 & 91 & 77.8 & \\
\hline \multicolumn{6}{|l|}{$\mathrm{HBeAg}$} \\
\hline Negative & 7 & 11.5 & 8 & 6.8 & 0.394 \\
\hline Positive & 54 & 88.5 & 109 & 93.2 & \\
\hline \multicolumn{6}{|l|}{ GGT } \\
\hline Negative & 35 & 57.4 & 63 & 53.8 & 0.751 \\
\hline Positive & 26 & 42.6 & 54 & 46.2 & \\
\hline \multicolumn{6}{|l|}{ Cirrhosis } \\
\hline Absent & 48 & 78.7 & 91 & 77.8 & 1.000 \\
\hline Present & 13 & 21.3 & 26 & 22.2 & \\
\hline \multicolumn{6}{|l|}{ AFP } \\
\hline$\leq 400 \mathrm{ng} / \mathrm{ml}$ & 40 & 65.6 & 79 & 67.5 & 0.867 \\
\hline$>400 \mathrm{ng} / \mathrm{ml}$ & 21 & 34.4 & 38 & 32.5 & \\
\hline \multicolumn{6}{|l|}{ Tumor size } \\
\hline$\leq 5 \mathrm{~cm}$ & 42 & 68.9 & 67 & 57.3 & 0.147 \\
\hline$>5 \mathrm{~cm}$ & 19 & 31.1 & 50 & 42.7 & \\
\hline \multicolumn{6}{|l|}{ No. of tumor nodules } \\
\hline Single & 48 & 78.7 & 100 & 85.5 & 0.293 \\
\hline Multiple & 13 & 21.3 & 17 & 14.5 & \\
\hline \multicolumn{6}{|l|}{ Tumor capsule } \\
\hline Well-differentiated & 33 & 54.1 & 62 & 53.0 & 1.000 \\
\hline Poorly differentiated & 28 & 45.9 & 55 & 47.0 & \\
\hline \multicolumn{6}{|l|}{ Microvascular invasion } \\
\hline Negative & 36 & 59.0 & 81 & 69.2 & 0.186 \\
\hline Positive & 25 & 41.0 & 36 & 30.8 & \\
\hline \multicolumn{6}{|c|}{ Tumor differentiation stage } \\
\hline I-II & 42 & 68.9 & 86 & 73.5 & 0.598 \\
\hline III-IV & 19 & 31.1 & 31 & 26.5 & \\
\hline \multicolumn{6}{|l|}{ Clinical stage } \\
\hline I & 45 & 73.8 & 80 & 68.4 & 0.494 \\
\hline II & 16 & 26.2 & 37 & 31.7 & \\
\hline \multicolumn{6}{|l|}{ UICC TNM stage } \\
\hline Low (stage I/II) & 53 & 86.9 & 105 & 89.7 & 0.620 \\
\hline High (stage III) & 8 & 13.1 & 12 & 10.3 & \\
\hline
\end{tabular}

AFP, $\alpha$-fetoprotein; GGT, $\gamma$-glutamyltransferase; HBeAg, hepatitis B e antigen; HCC, hepatocellular carcinoma; TNM, TNM classification of malignant tumors; UICC, Union for International Cancer Control. Pearson $\chi^{2}$ test. 
$10 \% \mathrm{FBS}$ and cultured in a 6-well plate at $37^{\circ} \mathrm{C}$ with $5 \% \mathrm{CO}_{2}$. After 14 days, colonies were photographed and counted. All experiments were performed in triplicate.

Invasion assay. The invasive ability of HCC cells was detected by 24 -well transwell chambers with $8-\mu \mathrm{m}$ pores of polycarbonate membranes (Costar, Cambridge, MA, USA) coated with Matrigel (BD Pharmingen, San Jose, CA, USA). The upper chamber was filled with FBS-free DMEM medium and the bottom chamber was filled with DMEM containing $10 \%$ FBS as a chemoattractant. Upper chambers containing cells $\left(5 \times 10^{4}\right)$ were filled and incubated at $37^{\circ} \mathrm{C}$. After $48 \mathrm{~h}$, cells migrating to the bottom of the membrane were stained with Giemsa (Sigma Chemical, St. Louis, MO, USA). Then, the cells were imaged and counted with a microscope (Leica, UK). All experiments were performed in triplicate.

Immunofluorescence assay. Cells on the cover slips were fixed with $4 \%$ paraformaldehyde for $30 \mathrm{~min}$ and permeabilized in $1 \%$ Triton X-100 for $15 \mathrm{~min}$ at room temperature. After blocking with $1 \%$ bovine serum albumin for $30 \mathrm{~min}$, the cells were incubated with primary antibodies for $4 \mathrm{~h}$. The following antibodies were used: human anti-E-cadherin (mouse monoclonal antibody, 1:50; Santa Cruz), Vimentin (mouse monoclonal antibody, 1:50; Santa Cruz), and human anti-MST4 (rabbit, polyclonal, 1:100; Proteintech). Then, samples were incubated with Alexa 488-conjugated and 594-conjugated secondary antibodies (Invitrogen) for $1 \mathrm{~h}$ at room temperature. Slides were mounted in medium containing DAPI (Vector Laboratories, Burlingame, CA, USA) and analyzed under a fluorescence microscope.

Western blotting. The proteins were extracted with RIPA buffer containing protease inhibitors, separated on a denaturing 10\% SDS-polyacrylamide gel, and transferred onto polyvinylidene difluoride membrane. After blocking with $5 \%$ non-fat milk in phosphate-buffered saline containing $0.05 \%$ Tween-20, the membrane was incubated with primary antibodies overnight at $4^{\circ} \mathrm{C}$, followed by incubating with peroxidase secondary antibodies for $1 \mathrm{~h}$ at $37^{\circ} \mathrm{C}$. The following antibodies were used: anti-human MST4 (rabbit, polyclonal, 1:500; Proteintech); anti-human GAPDH (rabbit, polyclonal, 1:1000; Cell Signaling Technology); anti-human p-ERK, ERK, E-cadherin, N-cadherin, Vimentin, Snail, and Slug, and the secondary antibody belonging to the EMT Antibody Sampler kit (rabbit, polyclonal, 1:1,000; Cell Signaling Technology). Western blotting was detected and analyzed with Image Acquisition using Image Quant LAS 4000 (GE Healthcare Life Sciences, MI, USA) and Quantity One software (Bio-Rad Laboratories), respectively.

Statistical analysis. Statistical analysis was performed with SPSS 13.0 (SPSS, Chicago, IL, USA). All data are presented as the mean \pm standard deviation. The Student's t-test and one-way analysis of variance were applied to analyze the difference of two groups or more than two groups, respectively. Kaplan-Meier analysis was used for survival analysis and log-rank test was used to compare patient survival between subgroups. A Cox proportional hazards model was adopted for multivariate analysis. Receiver-operating characteristic (ROC) curve analysis was applied to assess the predictive values of variables. $\mathrm{P}<0.05$ was considered statistically significant for all tests.

\section{Results}

High expression of MST4 in HCC cell lines and specimens with invasive and metastatic potential. Our previous cDNA array study showed that MST4 had higher expression level in HCC with metastasis than those without metastasis (22), indicating the correlation between MST4 level and metastatic potential of HCC. In this study, we first examined the protein expression of MST4 in the normal liver cell line and HCC cell lines with different metastatic abilities. Western blot analysis showed that MST4 was detected in higher levels in HCC cell lines with high metastatic potential (MHCC97H and MHCCLM3) than those cell lines with low-metastatic potential (Hep3B, PLC, and SMMC7721) and normal liver cell line L02 (Fig. 1A)

We then tested the mRNA and protein levels of MST4 in HCC samples with vascular invasion (VIHCC) and with non-vascular invasion (non-VIHCC), and found that they were much higher in VIHCC than that in non-VIHCC $(\mathrm{P}<0.05)$ (Fig. 1B and C), which is in agreement with our previous cDNA array results. Immunohistochemistry staining showed that MST4 expression was negative in normal and peritumoral liver tissue, relatively positive in non-VIHCC, and strongly positive in VIHCC (Fig. 1D). These results indicate that high expression of MST4 is closely associated with aggressive metastasis in HCC.

High MST4 protein expression in HCC is associated with worse clinical outcomes. We then evaluated the association of MST4 expression and the outcomes of 178 HCC patients with 5-year follow-up after operation using immunostaining and tissue microarrays. The clinicopathologic characteristics of the patients are shown in Table I. MST4 staining was mainly found in the cytoplasm of tumor cells and around the nucleus but was absent in most stromal cells (Fig. 2A). According to the HCC staining scores, all the samples were stratified into two grades: strong and mild MST4-positive staining were defined as high MST4 expression ( $\mathrm{n}=61)$, and low MST4positive staining and MST4-negative staining were defined as low MST4 expression ( $\mathrm{n}=117)$.

Statistical analysis showed that the high MST4 expression group displayed significantly worse overall survival (OS) (median OS, 58 months versus $>63$ months; log-rank=8.12; $\mathrm{P}=0.004)$ (Fig. 2B) and shortened time to tumor recurrence (TTR) (median TTR, 43.0 months versus $>63$ months; log-rank 10.31; $\mathrm{P}=0.001$ ) (Fig. 2C) compared to the low MST4 expression group. Large tumor size, microvascular invasion, multiple tumors, and advanced TNM classification of malignant tumors stage were found to be associated with worse OS and shortened TTR in univariate analysis. Multivariate analysis results revealed that MST4 intensity in tumors is an independent prognosticator for both OS (relative risk, 1.863; $\mathrm{P}=0.012$ ) and TTR (relative risk, 2.348; $\mathrm{P}=0.001$ ) (Table II), suggesting that MST4 is a valuable predictor of clinical outcomes in HCC patients.

MST4 enhanced proliferation and invasive potential of HCC cells in vitro. Considering the close association of 

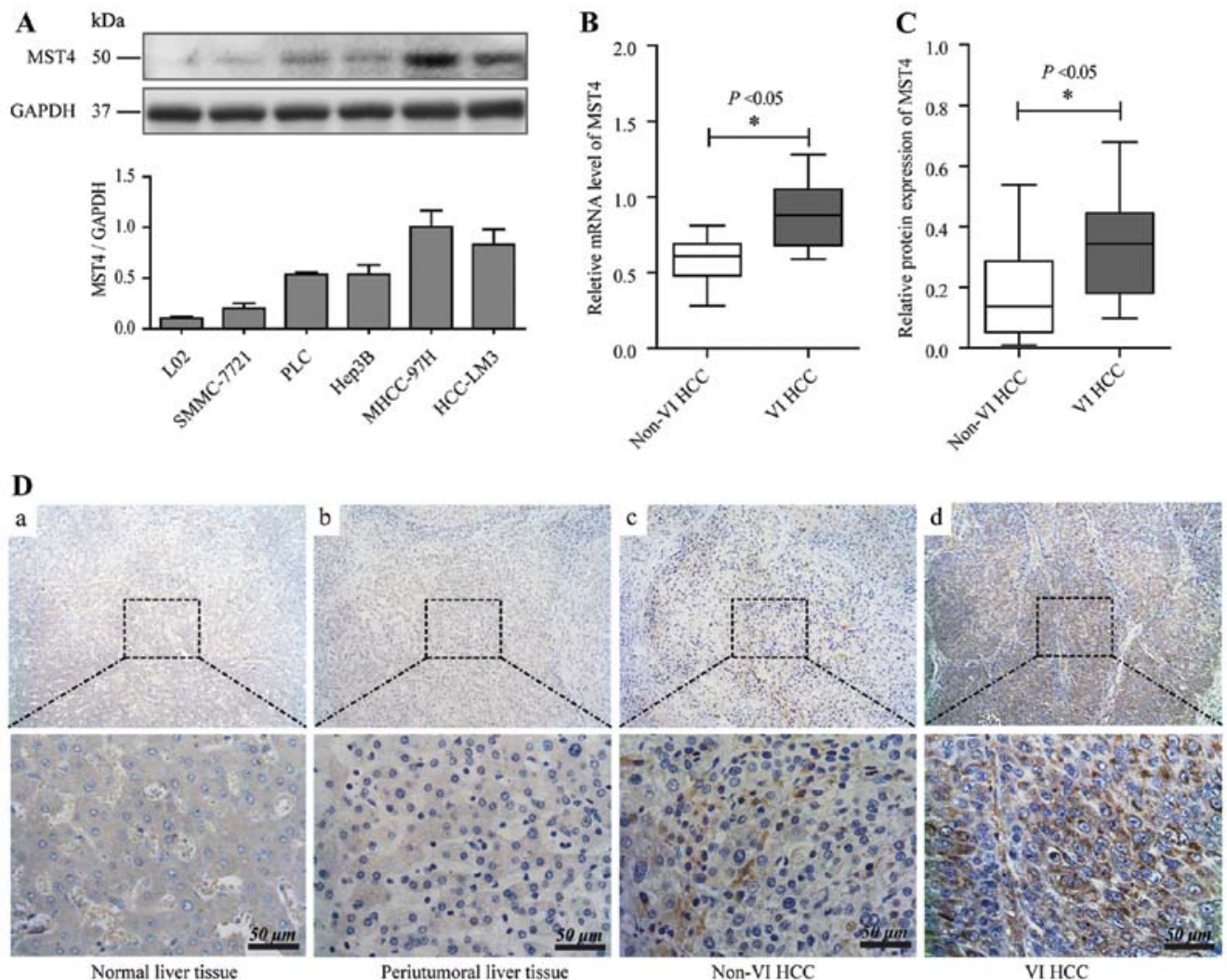

Figure 1. Increased MST4 protein expression in HCC cell lines with metastatic potential and in invasive HCC specimens. MST4 expression was evaluated in the indicated HCC cell lines and HCC specimens. High MST4 expression was found in cells with high potential for malignancy (A). Quantitative reversetranscription polymerase chain reaction (B) and western blotting (C) data showed that the MST4 level was significantly higher in HCC with vascular invasion (VIHCC) than in HCC without vascular invasion (non-VIHCC). HCC specimens (D) in a tissue microarray were immunostained. Representative images are shown for normal liver tissue, peritumoral liver tissue, non-VIHCC and VIHCC (magnification, upper panels, x100; lower panels, x400).
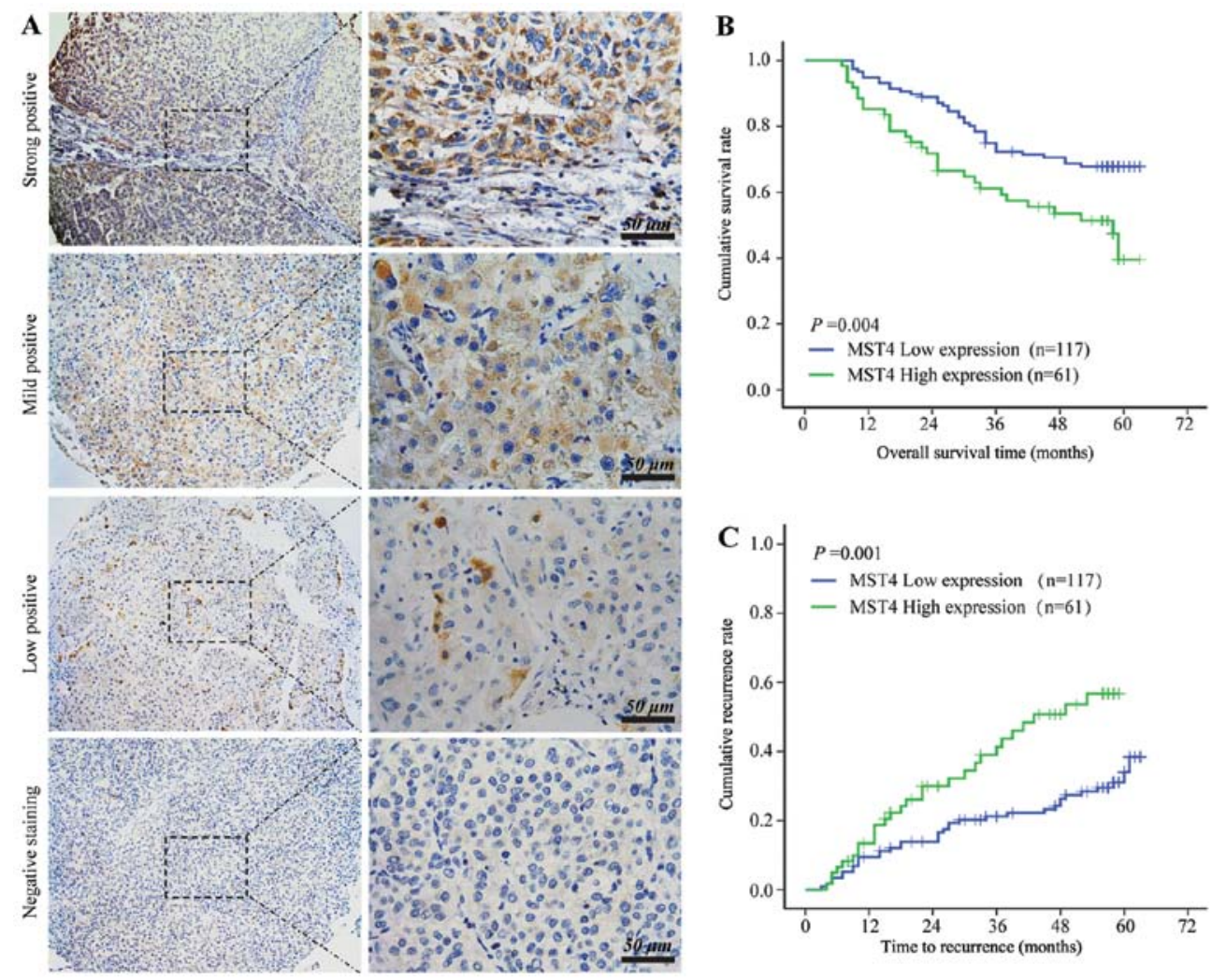

Figure 2. High MST4 protein expression in HCC is associated with worse clinical outcomes. MST4 staining was mainly found in the cytoplasm of tumor cells and around the nucleus but was absent in the most stromal cells. (A) The staining was divided into four grades: negative; low-positive; mildly positive; and strongly positive. Significant differences appear in OS (B) and TTR (C) in postoperative HCC patients with high and low MST4 levels in primary HCC tissues. 
Table II. Univariate and multivariate analysis of factors associated with survival and recurrence.

\begin{tabular}{|c|c|c|c|c|c|c|c|c|}
\hline \multirow[b]{3}{*}{ Features } & \multicolumn{4}{|c|}{ Overall survival } & \multicolumn{4}{|c|}{ Time to recurrence } \\
\hline & \multirow{2}{*}{$\frac{\text { Univariate }}{\text { P-value }}$} & \multicolumn{3}{|c|}{ Multivariate } & \multirow{2}{*}{$\frac{\text { Univariate }}{\text { P-value }}$} & \multicolumn{3}{|c|}{ Multivariate } \\
\hline & & HR & $95 \% \mathrm{CI}$ & P-value & & HR & $95 \% \mathrm{CI}$ & P-value \\
\hline \multicolumn{9}{|l|}{ Age } \\
\hline$\leq 50$ vs. $>50$ years & 0.967 & & & NA & 0.545 & & & NA \\
\hline \multicolumn{9}{|l|}{ Sex } \\
\hline Female vs. male & 0.771 & & & NA & 0.983 & & & NA \\
\hline \multicolumn{9}{|l|}{ Hepatitis B antigen } \\
\hline Negative vs. positive & 0.471 & & & NA & 0.869 & & & NA \\
\hline \multicolumn{9}{|l|}{ Liver cirrhosis } \\
\hline No vs. yes & 0.291 & & & NA & 0.033 & 3.074 & $1.223-7.725$ & 0.017 \\
\hline \multicolumn{9}{|l|}{ AFP } \\
\hline$\leq 400$ vs. $>400 \mathrm{ng} / \mathrm{ml}$ & 0.093 & & & NA & 0.791 & & & NA \\
\hline \multicolumn{9}{|l|}{ Tumor size } \\
\hline$\leq 5 \mathrm{vs} .>5 \mathrm{~cm}$ & 0.003 & 1.734 & $1.028-2.924$ & 0.039 & 0.013 & & & NS \\
\hline \multicolumn{9}{|l|}{ No. of tumor } \\
\hline Single vs. multiple & 0.052 & & & NA & 0.010 & & & NS \\
\hline \multicolumn{9}{|l|}{ Tumor encapsulation } \\
\hline None vs. complete & 0.336 & & & NA & 0.067 & & & NA \\
\hline \multicolumn{9}{|l|}{ Microvascular invasion } \\
\hline No vs. yes & 0.004 & & & NS & 0.027 & & & NS \\
\hline \multicolumn{9}{|l|}{ Tumor differentiation stage } \\
\hline I-II vs. III-IV & 0.063 & & & NA & 0.815 & & & NA \\
\hline \multicolumn{9}{|l|}{ TNM stage } \\
\hline I vs. II vs. IIIa & 0.006 & 1.631 & $1.164-2.285$ & 0.004 & 0.001 & 1.692 & $1.209-2.368$ & 0.002 \\
\hline \multicolumn{9}{|l|}{ MST4 } \\
\hline High vs. low expression & 0.004 & 1.863 & $1.148-3.023$ & 0.012 & 0.001 & 2.348 & $1.405-3.924$ & 0.001 \\
\hline \multicolumn{9}{|l|}{ p-ERK } \\
\hline High vs. low expression & 0.038 & & & NS & 0.029 & 2.363 & $1.013-2.758$ & 0.044 \\
\hline Both MST4 and p-Erk & 0.001 & & & NA & $<0.001$ & & & NA \\
\hline
\end{tabular}

AFP, $\alpha$-fetoprotein; CI, confidence interval; HR, hazard ratio; MST4, mammalian sterile-20-like kinase 4; NA, not adopted; NS, non-significant; p-ERK, phosphorylated extracellular signal-regulated protein kinase; TNM, TNM classification of malignant tumors. Univariate analysis, Cox proportional hazards regression model.

high MST4 expression and high recurrence rate of HCC, we examined whether high MST4 level plays a key role in HCC proliferation and invasion. To verify this inference, lentivirus-mediated knockdown in highly invasive MHCC-97H cells was performed to assess the functional involvement of MST4 in HCC cell proliferation, colony formation, and invasion in vitro. We generated three MST4specific shRNAs (shMST4) and infected MHCC-97H cells with a lentivirus expressing shMST4 precursor, and found the second group of shMST4 induced $>80 \%$ reduction which was used for further study (Fig. 3A, left panel). Compared to the scrambled shRNA group (SCR group) and wild-type group (WT group), the proliferation and the clone formation in the MST4 knockdown group (KD group) were restrained significantly $(\mathrm{P}<0.01)$ (Fig. 3B, left panel and Fig. 3C, upper panel). Furthermore, depletion of MST4 also led to dramatic decline in invasiveness potential (transwell assay, $\mathrm{P}<0.01$ ) (Fig. 3D, lower panel). We generated the SMCC7721 clone with stable lentivirus-mediated overexpression of MST4 (MST4 group) and the SMCC7721 empty vector as the control group (MOCK group) and found that exogenous expression of MST4 in the MST4 group significantly enhanced HCC cell proliferation, 
A
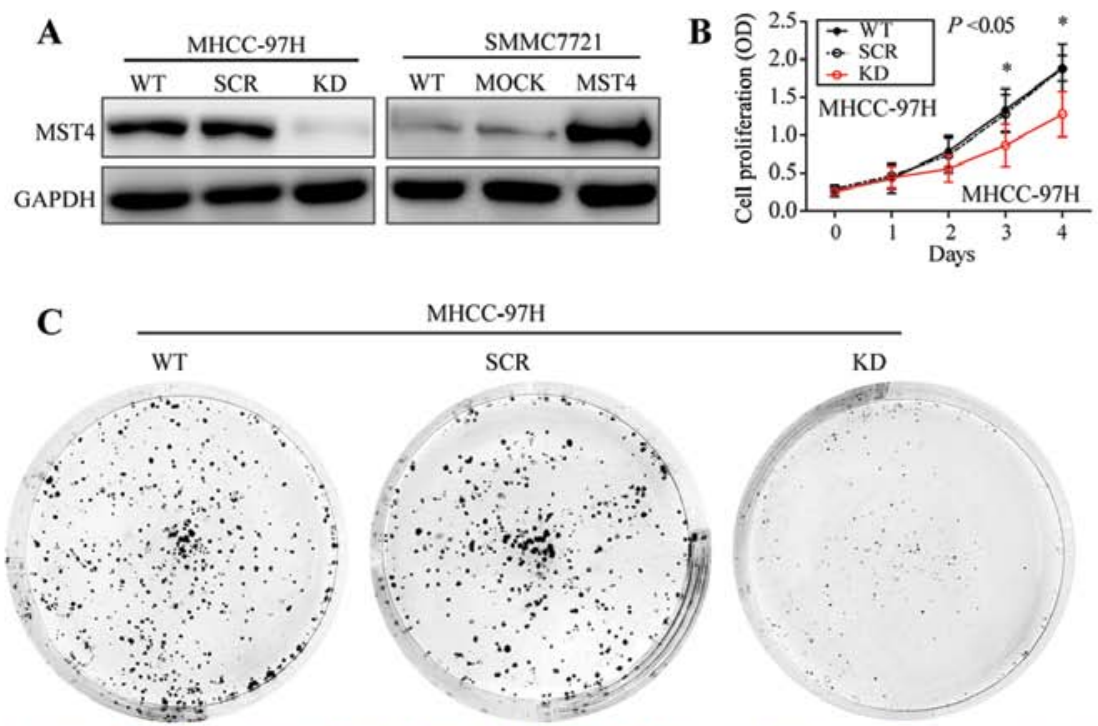

MHCC-97H
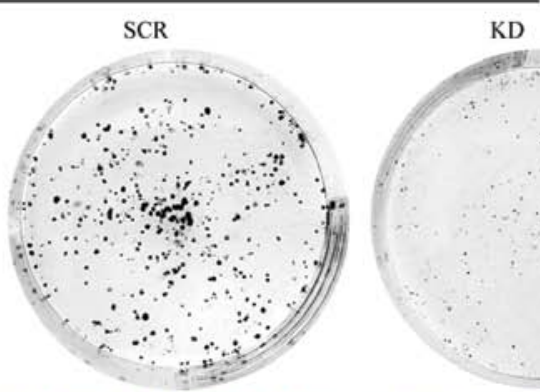

KD
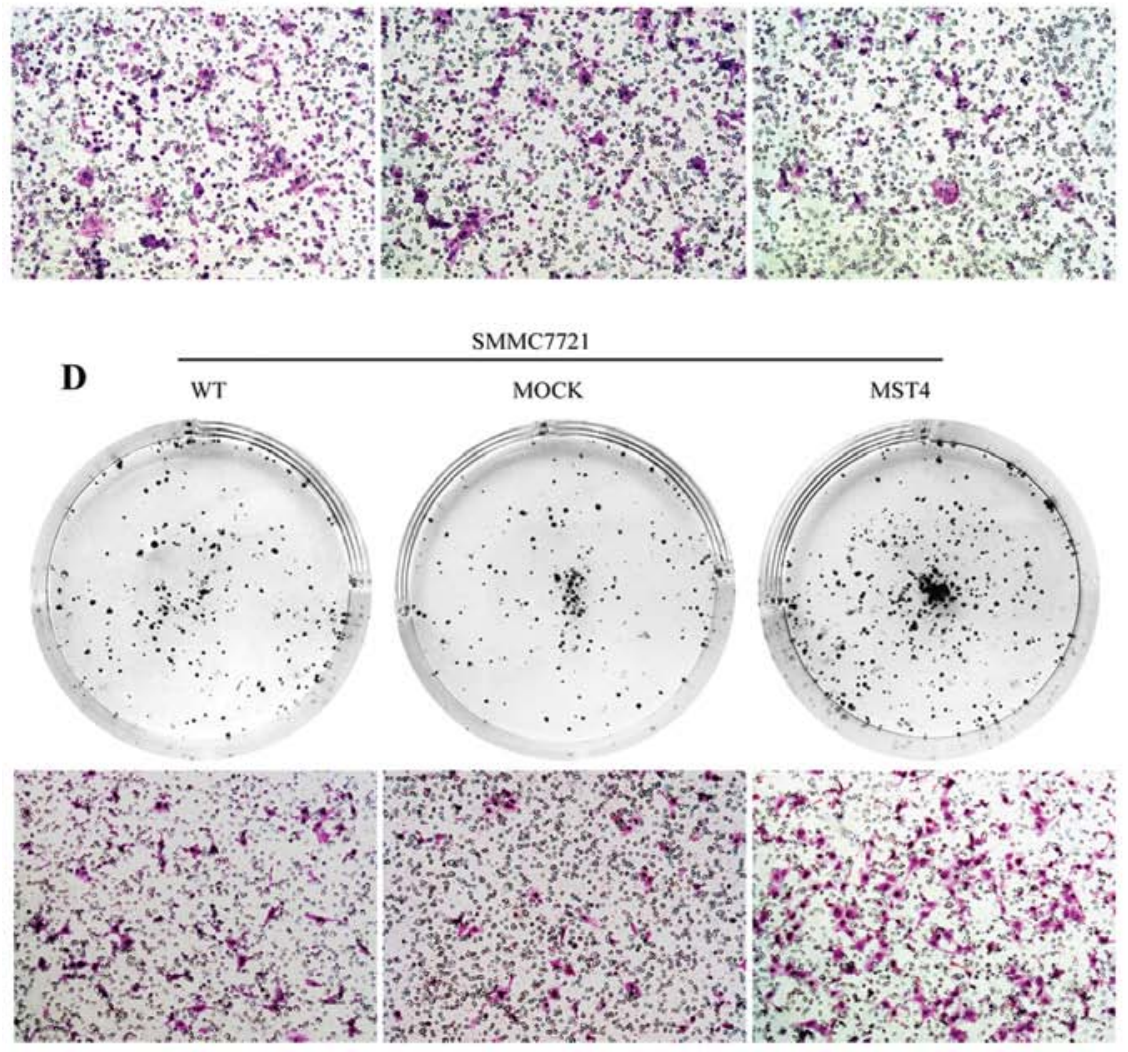
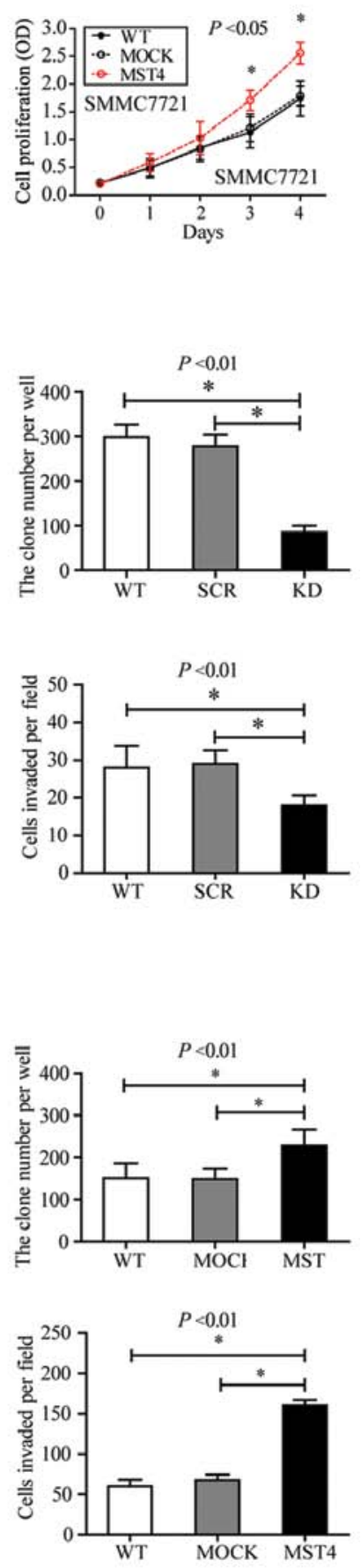

Figure 3. MST4 enhances proliferation and invasive potential of HCC cells in vitro. Compared with the wild-type group (WT) and the scrambled shRNA group (SCR), lentivirus-mediated shRNA resulted in the significant downregulation of MST4 in MHCC-97H cells (A, left panel). Overexpression of MST4 occurred in SMMC7721 cells (A, right panel). Knockdown of MST4 in MHCC-97H cells inhibited proliferation (B), colony formation C), and invasion (C), and overexpression of MST4 in SMMC7721 cells promoted these processes (B and D).

colony formation, and invasion compared with wild-type and mock groups(Fig. 3). Taken together, these results suggest that MST4 plays an important role in HCC proliferation and invasion.

MST4 promotes mesenchymal transformation in HCC cells. EMT is an important process in tumor proliferation and invasion. To investigate whether MST4 altered the EMT process in HCC metastatic progression, we examined the expression level of E-cadherin, $\mathrm{N}$-cadherin, and Vimentin, the cell markers of EMT, in HCC cells. As shown in Fig. 4A and C, relative to the scrambled shRNA group (SCR group), decreased expression of MST4 (KD group) resulted in upregulation of E-cadherin and downregulation of $\mathrm{N}$-cadherin and Vimentin, whereas overexpression of MST4 (MST4 group) led to downregulation of E-cadherin and upregulation of $\mathrm{N}$-cadherin and Vimentin, compared to the control group (MOCK group). Immunofluorescence staining also confirmed these results in Fig. 4B and D. The expression of E-cadherin is negatively regulated by transcription factors, such as Snail and Slug (24). As 
A

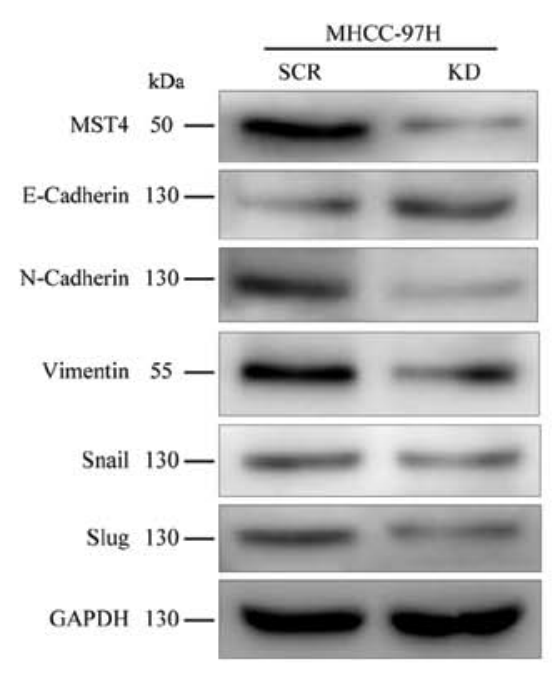

C

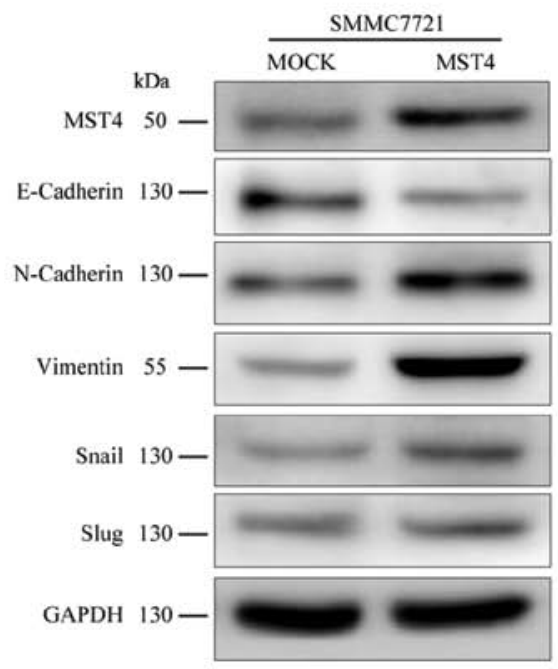

B

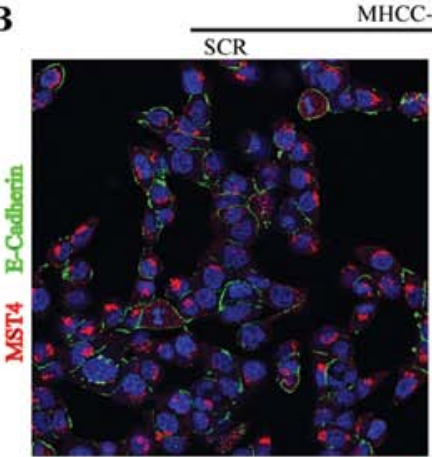

MHCC-97H
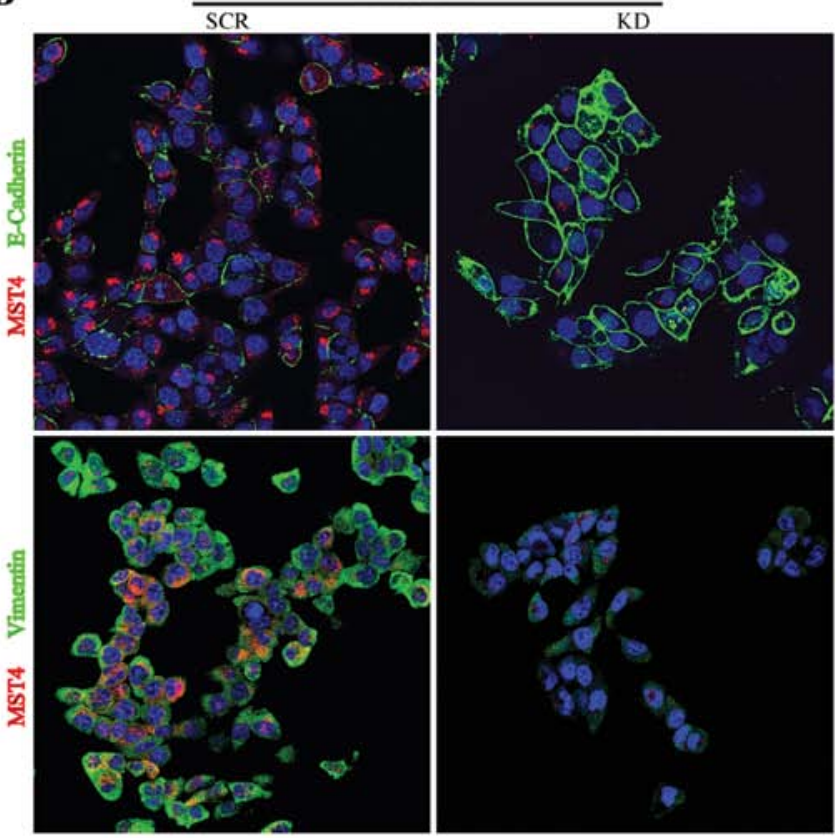

SMMC7721

D
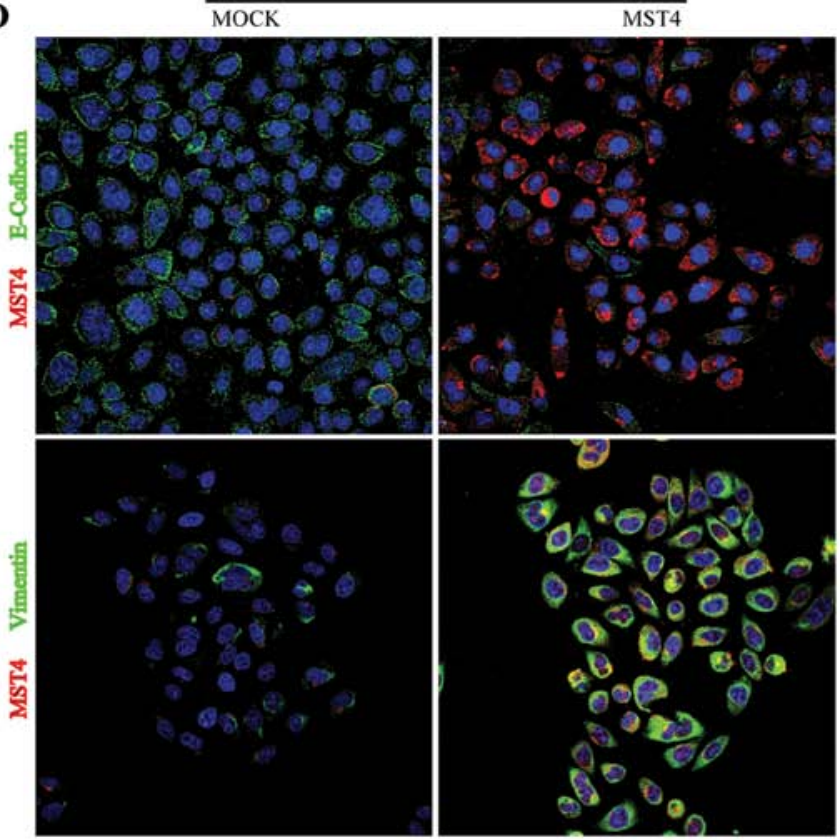

Figure 4. MST4 promotes EMT in HCC cells. Knockdown of MST4 expression in MHCC-97H cells increased E-cadherin expression and reduced N-cadherin, Vimentin, Snail, and Slug expression, as shown with western blotting (A) and fluorescent microscopy (B). Nuclei are stained with DAPI. Overexpression of MST4 in SMMC7721 cells reduced E-cadherin expression and increased N-cadherin, Vimentin, Snail, and Slug expression, as shown with western blotting (C) and fluorescent microscopy (D).

illustrated in Fig. 4A and C, the levels of Snail and Slug were dramatically downregulated in the MHCC-97H knockdown group and significantly upregulated after MST4 overexpression in SMCC7721. These results indicate that MST4 may promote malignant progression of $\mathrm{HCC}$ by inducing the EMT process.

ERK pathway plays a critical role in mediating MST4 function. Mounting evidence indicates that Snail and Slug play crucial roles in initiating EMT process $(25,26)$, and they are both important downstream targets of the ERK signaling pathway $(27,28)$. To further investigate the mechanisms of MST4 in promoting EMT process in HCC cells, we examined the influence of MST4 overexpression on the ERK pathway.

HCC cell lines SMMC7721 and Hep3B with MST4 overexpression were generated with stable lentivirus-mediated MST4 (MST4 group) and the empty vector (control MOCK group). As shown in Fig. 5A and B, the ratio of p-ERK to ERK was upregulated in the MST4 group compared to the mock group. This trend was similar to that of the mesenchymal markers 

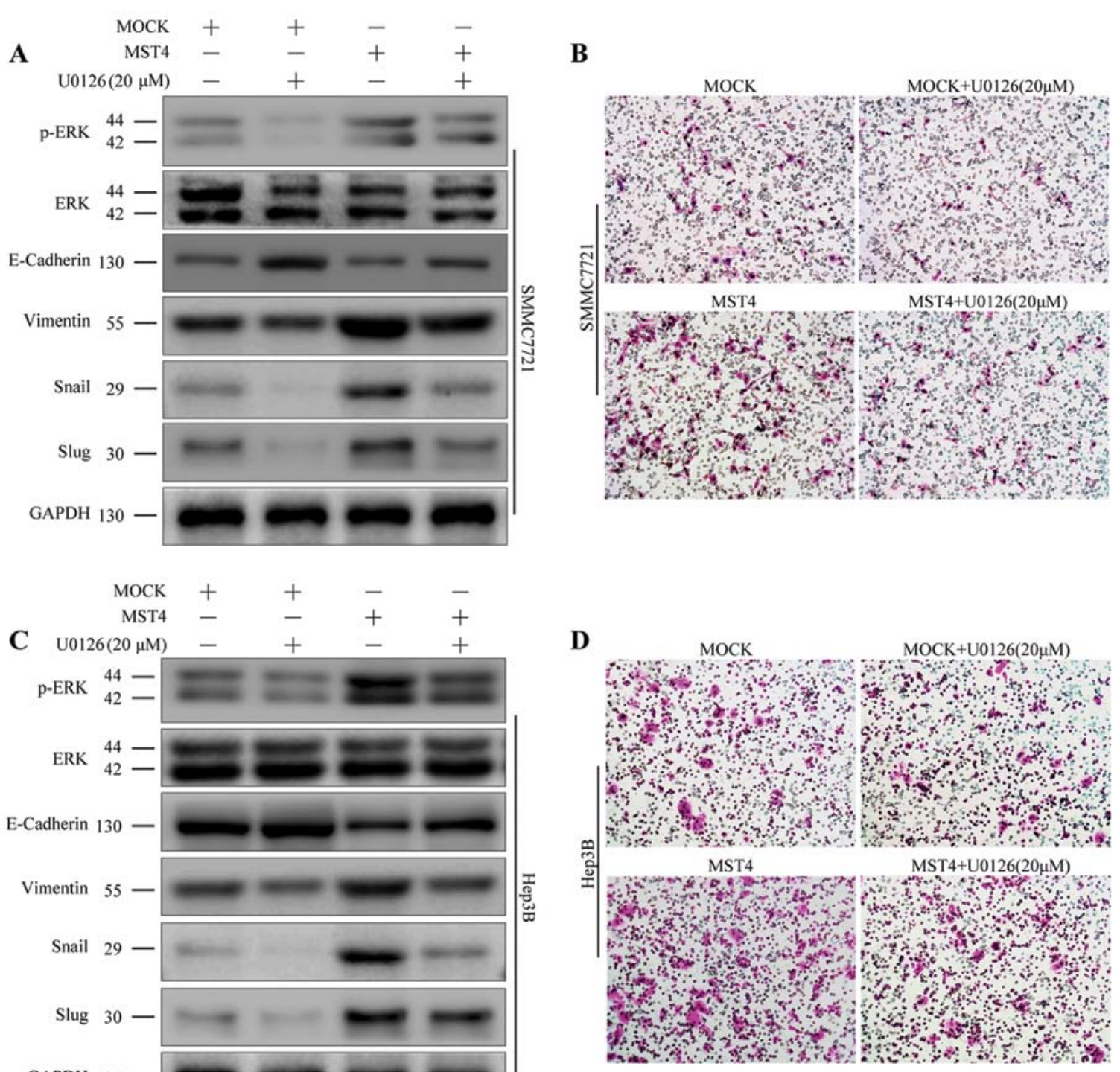

Figure 5. The ERK pathway plays a critical role in mediating MST4 function. In SMCC7721 cells, overexpression of MST4 activated the p-ERK pathway with high expression of Vimentin, Snail, and Slug and with low expression of E-cadherin, whereas U0126 $(20 \mu \mathrm{M})$, the inhibitor of p-ERK, partly diminished MST4-induced EMT (A) with low invasiveness (B). Similar results were found in Hep3B cells (C and D).

such as Snail, Slug, and Vimentin, but was contrary to that of E-cadherin.

To further confirm the key role of the p-ERK/ERK pathway in the promotion of EMT with MST4, we used the p-ERK inhibitor (U0126, $20 \mu \mathrm{M}$; Sigma-Aldrich) to decrease the activation of ERK. As shown in Fig. 5, U0126 treatment partially reversed the MST4-induced increase in the ratio of p-ERK to ERK and the mesenchymal markers but did not bring it back down to control levels. In addition, U0126 treatment also suppressed the migratory and invasive behavior of the SMMC7721 and Hep3B cells with MST4 overexpression (Fig. 5B and D). These data revealed that overexpression of MST4 promote the EMT process, at least in part, through upregulation of the ERK signaling pathway.

Combination of MST4 and p-ERK levels has a better prognostic value for HCC. On the basis of the in vitro finding that MST4 regulated phosphorylation of ERK in HCC, we further investigated the relationship between MST4 and p-ERK in HCC tissue microarray. Positive p-ERK staining was partly detected in cytoplasmic and nuclear HCC cells (Fig. 6A), and p-ERK expression was significantly correlated with MST4 expression (data not shown). To further confirm the prognostic value of MST4 and p-ERK, HCC tissues were classified into the following three groups according to the positive staining value of MST4 and p-ERK: group $1(n=67)$, low MST4 and low p-ERK expression; group $2(\mathrm{n}=81)$, either high MST4 or high p-ERK expression; and group $3(n=30)$, high MST4 and high $\mathrm{p}$-ERK expression. There were significant differences in OS $(\mathrm{P}=0.001)$ (Fig. 6B) and TTR $(\mathrm{P}<0.001)$ (Fig. 6C) among the three groups.

Multivariate survival analysis results showed that MST4 alone could predict death and recurrence precisely with the areas under the receiver-operating characteristic curve of 0.584 [95\% confidence interval (CI), 0.503-0.672] and 0.579 (95\% CI, 0.502-0.668), respectively. The combination of MST4 

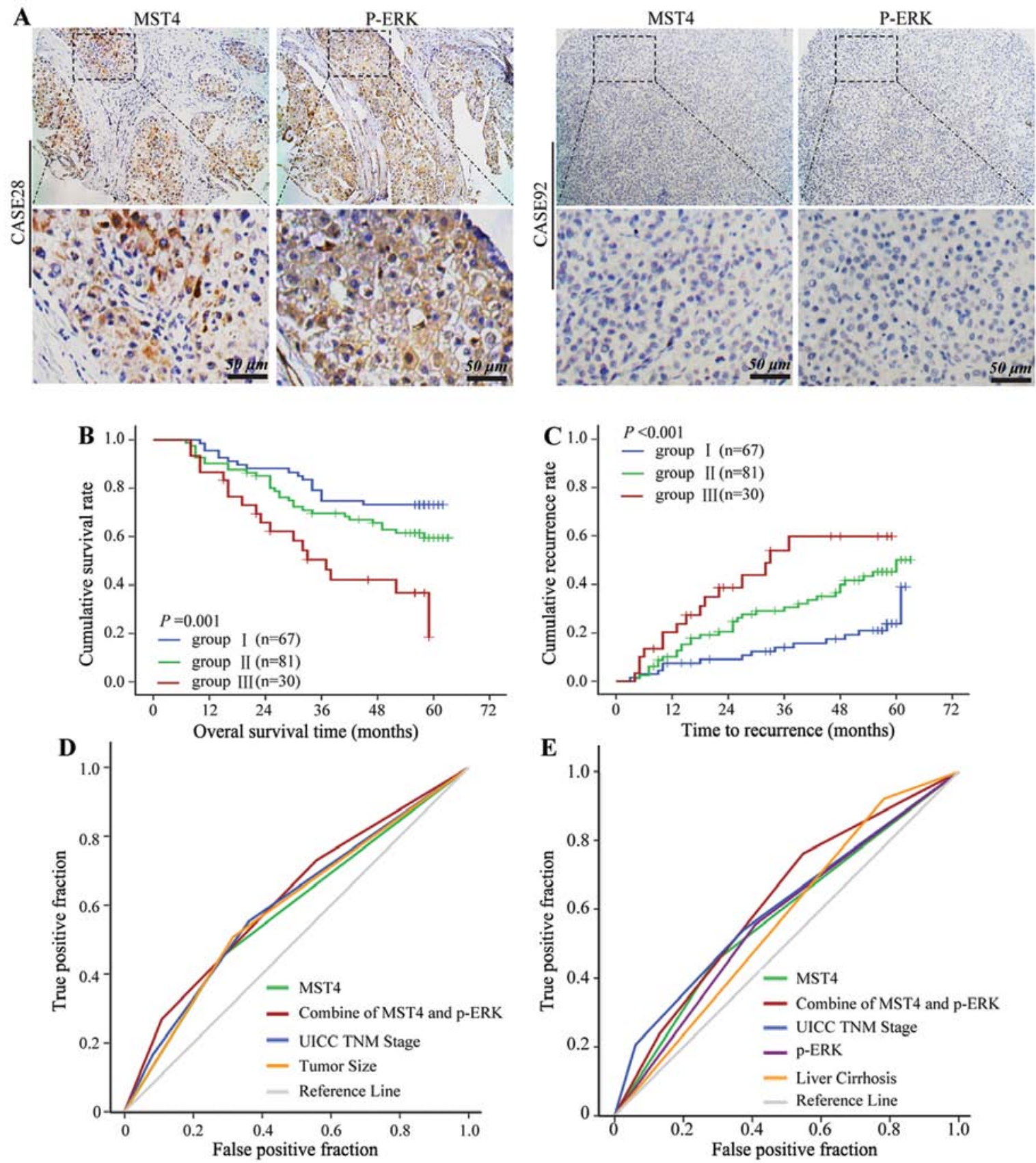

Figure 6. Combination of MST4 and p-ERK levels has a better prognostic value for HCC outcomes. The expression of HCC MST4 was associated with the expression of HCC p-ERK. Representative images are shown (A). The cumulative OS (B) and TTR (C) curves of the combination of HCC MST4 and p-ERK are also shown. All factors used in receiver-operating characteristic curve analysis predicted death (D) and recurrence (E) during follow-up.

and p-ERK further elevated the area under the curve and was better for predicting death $(0.622 ; 95 \% \mathrm{CI}, 0.532-0.707)$ (Fig. 6D) and recurrence (0.623; 95\% CI, 0.537-0.708) (Fig. 6E) for HCC.

\section{Discussion}

Currently, the molecular mechanism of HCC invasion and metastasis is still not well-understood, and the high rate of $\mathrm{HCC}$ metastatic recurrence is the main hindrance in further improvement of overall survival after hepatectomy. It is urgent to find new innovative therapeutic targets to improve HCC prognosis. MST4, a member of the protein family that shares similarity with Ste20, is an MST kinase (12,16-18), which has been found to play important roles in regulating multiple cell functions, such as cell polarity and proliferation $(9,10)$, but little is known about its oncogenic role in HCC progression. In our previous gene expression profile study of HCC without or with intrahepatic metastasis, MST4 was identified as an important candidate gene for HCC metastasis (22). Here we elucidated that high MST4 expression was associated with malignance in HCC cell lines with different metastatic potential, and in vitro experiments found that MST4 played important roles in proliferation, colony formation, and invasion in HCC cells. We 
also verified that high expression of MST4 in HCC was associated with a high incidence of intrahepatic metastasis, shorter time to progression, and poor survival after hepatectomy, and univariate and multivariate analysis results revealed that MST4 is an independent prognostic indicator for both OS and TTR in HCC patients. These results suggest that MST4 plays important roles in HCC proliferation, invasion and metastasis.

Mounting evidence has indicated that EMT is an important process for tumor invasion and metastasis. In this study, downregulation of MST4 expression resulted in a sharp decline in proliferation, colony formation, and invasion of the HCC cells with high expression of E-cadherin and low expression of $\mathrm{N}$-cadherin and Vimentin. Conversely, overexpression of MST4 promoted the metastatic potential of the HCC cells with accumulation of $\mathrm{N}$-cadherin and Vimentin and decrease of E-cadherin. Considering the crucial role of EMT in the progression and metastasis of multiple cancers and the influence of the expression of MST4 accompanied by the change in mesenchymal marker Vimentin and epithelial marker E-cadherin and N-cadherin, we proposed that MST4 is a key protein in promoting the EMT process and is a promising molecule in shaping the postoperative strategy for prevention of metastatic recurrence for HCC.

Moreover, immunofluorescence staining confirmed that the expression of E-cadherin was negatively regulated by transcription factors Snail and Slug. The levels of Snail and Slug were dramatically downregulated in the MST4 knockdown group and significantly upregulated after MST4 overexpression. It is well known that p-ERK/ERK signaling pathways upregulate Snail and Slug, thereby leading to the downregulation of epithelial marker E-cadherin $(29,30)$. There is also evidence implying that MST4 influences cell growth and transformation by modulating a ras/rafindependent ERK pathway (16). We observed in our study that exogenous expression of MST4 in SMMC7721 and Hep3B cells enhanced cell proliferation and invasion with hyperactivation of ERK signaling pathways. The inhibition of ERK signaling pathways, through addition of specific inhibitor U0126, significantly suppressed the expression of Snail and Slug without affecting MST4 expression levels and blocked MST4-mediated promotion of migration and invasion. Moreover, the combination of MST4 and p-ERK in receiver-operating characteristic curve analysis had better prognostic value than MST4 alone for OS and accumulated recurrence for HCC. These findings demonstrate that MST4 promotes EMT, at least in part, through upregulation of the ERK signaling pathways, and inhibitors of p-ERK might be a potent therapeutic approach against HCC metastasis.

In conclusion, our results indicate that high expression of MST4 is a major contributor to the invasive phenotype of HCC by promoting the EMT process, and MST4 alone or combination with p-ERK is a novel prognostic marker and potential therapeutic target for $\mathrm{HCC}$.

\section{Acknowledgements}

We thank Mr. Wei-De Zhang for assistance in collecting patient data. This study was jointly supported by two grants (nos. 81071993 and 81372654) from the National Natural Science Foundation of China.

\section{References}

1. Ferlay J, Shin HR, Bray F, Forman D, Mathers C and Parkin DM: Estimates of worldwide burden of cancer in 2008: GLOBOCAN 2008. Int J Cancer 127: 2893-2917, 2010.

2. Jemal A, Bray F, Center MM, Ferlay J, Ward E and Forman D: Global cancer statistics. CA Cancer J Clin 61: 69-90, 2011.

3. Portolani N, Coniglio A, Ghidoni S, et al: Early and late recurrence after liver resection for hepatocellular carcinoma: prognostic and therapeutic implications. Ann Surg 243: 229-235, 2006.

4. Liu Y, Zhang JB, Qin Y, et al: PROX1 promotes hepatocellular carcinoma metastasis by way of up-regulating hypoxia-inducible factor 1alpha expression and protein stability. Hepatology 58: 692-705, 2013.

5. Turley EA, Veiseh M, Radisky DC and Bissell MJ: Mechanisms of disease: epithelial-mesenchymal transition - does cellular plasticity fuel neoplastic progression? Nat Clin Pract Oncol 5: 280-290, 2008.

6. Kalluri R and Weinberg RA: The basics of epithelial-mesenchymal transition. J Clin Invest 119: 1420-1428, 2009.

7. Zeisberg $\mathrm{M}$ and Neilson EG: Biomarkers for epithelial-mesenchymal transitions. J Clin Invest 119: 1429-1437, 2009.

8. Tse JC and Kalluri R: Mechanisms of metastasis: epithelialto-mesenchymal transition and contribution of tumor microenvironment. J Cell Biochem 101: 816-829, 2007.

9. Shi Z, Jiao S, Zhang Z, et al: Structure of the MST4 in complex with MO25 provides insights into its activation mechanism. Structure 21: 449-461, 2013.

10. Ling P, Lu TJ, Yuan CJ and Lai MD: Biosignaling of mammalian Ste20-related kinases. Cell Signal 20: 1237-1247, 2008.

11. Delpire E: The mammalian family of sterile $20 \mathrm{p}$-like protein kinases. Pflugers Arch 458: 953-967, 2009.

12. Dan I, Watanabe NM and Kusumi A: The Ste20 group kinases as regulators of MAP kinase cascades. Trends Cell Biol 11: 220-230, 2001.

13. Hao W, Takano T, Guillemette J, Papillon J, Ren G and Cybulsky AV: Induction of apoptosis by the Ste20-like kinase SLK, a germinal center kinase that activates apoptosis signalregulating kinase and p38. J Biol Chem 281: 3075-3084, 2006.

14. Nicke B, Bastien J, Khanna SJ, et al: Involvement of MINK, a Ste20 family kinase, in Ras oncogene-induced growth arrest in human ovarian surface epithelial cells. Mol Cell 20: 673-685, 2005.

15. Record CJ, Chaikuad A, Rellos P, et al: Structural comparison of human mammalian ste20-like kinases. PLoS One 5: e11905, 2010.

16. Lin JL, Chen HC, Fang HI, Robinson D, Kung HJ and Shih HM: MST4, a new Ste20-related kinase that mediates cell growth and transformation via modulating ERK pathway. Oncogene 20: 6559-6569, 2001.

17. Dan I, Ong SE, Watanabe NM, et al: Cloning of MASK, a novel member of the mammalian germinal center kinase III subfamily, with apoptosis-inducing properties. J Biol Chem 277: 5929-5939, 2002.

18. Qian Z, Lin C, Espinosa R, LeBeau M and Rosner MR: Cloning and characterization of MST4, a novel Ste20-like kinase. J Biol Chem 276: 22439-22445, 2001.

19. MaX, Zhao H, Shan J, et al: PDCD10 interacts with Ste20-related kinase MST4 to promote cell growth and transformation via modulation of the ERK pathway. Mol Biol Cell 18: 1965-1978, 2007.

20. Sung V, Luo W, Qian D, Lee I, Jallal B and Gishizky M: The Ste20 kinase MST4 plays a role in prostate cancer progression. Cancer Res 63: 3356-3363, 2003.

21. Preisinger C, Short B, De Corte V, et al: YSK1 is activated by the Golgi matrix protein GM130 and plays a role in cell migration through its substrate 14-3-3zeta. J Cell Biol 164: 1009-1020, 2004.

22. Ye QH, Qin LX, Forgues M, et al: Predicting Hepatitis B virus-positive metastatic hepatocellular carcinomas using gene expression profiling and supervised machine learning. Nat Med 9: 416-423, 2003.

23. Zhu XD, Zhang JB, Zhuang PY, et al: High expression of macrophage colony-stimulating factor in peritumoral liver tissue is associated with poor survival after curative resection of hepatocellular carcinoma. J Clin Oncol 26: 2707-2716, 2008 . 
24. Batlle E, Sancho E, Franci C, et al: The transcription factor snail is a repressor of E-cadherin gene expression in epithelial tumour cells. Nat Cell Biol 2: 84-89, 2000.

25. Zhou BP, Deng J, Xia W, et al: Dual regulation of Snail by GSK-3beta-mediated phosphorylation in control of epithelialmesenchymal transition. Nat Cell Biol 6: 931-940, 2004.

26. Doble BW and Woodgett JR: Role of glycogen synthase kinase-3 in cell fate and epithelial-mesenchymal transitions. Cells Tissues Organs 185: 73-84, 2007.

27. Cross DA, Alessi DR, Cohen P, Andjelkovich Mand Hemmings BA Inhibition of glycogen synthase kinase- 3 by insulin mediated by protein kinase B. Nature 378: 785-789, 1995.
28. Cohen P and Frame S: The renaissance of GSK3. Nat Rev Mol Cell Biol 2: 769-776, 2001.

29. Hong KO, Kim JH, Hong JS, et al: Inhibition of Akt activity induces the mesenchymal-to-epithelial reverting transition with restoring E-cadherin expression in KB and KOSCC-25B oral squamous cell carcinoma cells. J Exp Clin Cancer Res 28: 28-39, 2009.

30. Conacci-Sorrell M, Simcha I, Ben-Yedidia T, Blechman J, Savagner P and Ben-Ze'Ev A: Autoregulation of E-cadherin expression by cadherin-cadherin interactions: the roles of betacatenin signaling, Slug, and MAPK. J Cell Biol 163: 847-857, 2003. 